\title{
Wahlkampf und Jugendliche: Nur Risiken und Nebenwirkungen? Ergebnisse einer internationalen Längsschnittstudie
}

\author{
Michael C. Hermann
}

Kein Wahlkampfmanager würde der Feststellung widersprechen, dass es die Aufgabe von Wahlkampf ist, Unterstützung der Wähler für die jeweilige Partei zu mobilisieren. Hierfür haben die Parteien ein breites Spektrum von Wahlkampfstrategien, -taktiken und -instrumenten entwickelt, die von einer expandierenden politischen Kommunikationswissenschaft eingeordnet und untersucht werden. ${ }^{1}$ Viel Aufmerksamkeit finden dabei aktuell die Modernisierungen von Wahlkampf, wie sie oft auch mit dem Begriff der Amerikanisierung ${ }^{2}$ umschrieben werden.

\section{Wirkungen von Wablkampf}

Weniger diskutiert wird die Frage, welche eher mittel- bis langfristigen Effekte Wahlkampf in modernen Demokratien hat. Dabei geht es um die Wirklichkeitskonstruktion von Politik, die durch Wahlkampf erzeugt wird. Dieses so entstandene Bild von Politik bestimmt nachhaltig die Legitimation, die die Bürger dem politisch-administrativen System entgegenbringen. Dies gilt unabhängig davon, ob es sich um richtige Vorstellungen oder um Fehlperzeptionen handelt. Diese Zusammenhänge werden immer wichtiger. Die allmähliche Erosion der Legitimation, die für die Stabilität einer Demokratie erforderlich ist, ist deutlich wahrnehmbar. Sie ist nicht nur sichtbar in der Diskussion um die so genannte Politikverdrossenheit ${ }^{3}$, sondern auch in rückläufiger Wahlbeteiligung auf allen

1 Vgl. Christina Holtz-Bacha, Strategien des modernen Wahlkampfes, in: APuZ, B 7 (2006), S. 11 - 19; dies. (Hrsg.), Die Massenmedien im Wahlkampf: die Bundestagswahl 2002, Wiesbaden 2003; David L. Swanson / Paolo Manicini, Patterns of Modern Electoral Campaigning and their Consequences, in: dies. (Hrsg.), Politics, Media and Modern Democracy. An International Study of Innovations in Electoral Campaigning and their Consequences, Westport / London 1996, S. 247 - 276; Frank Brettschneider, Die Medienwahl 2002: Themenmanagement und Berichterstattung, in: APuZ, B 49/50 (2002), S. 36 - 47; Fritz Plasser / Franz Sommer / Christian Scheucher, Medienlogik: Themenmanagement und Politikvermittlung im Wahlkampf, in: Fritz Plasser / Peter Ulram / Günther Ogris (Hrsg.), Wahlkampf und Wählerentscheidung. Analysen zur Nationalratswahl 1995, Wien 1996, S. 85 - 118; Elisabeth Noelle-Neumann / Hans Mathias Kepplinger / Wolfgang Donsbach (Hrsg.), Kampa. Meinungsklima und Medienwirkung im Bundestagswahlkampf 1998, Freiburg / München 1999.

2 „Die Akzente eines amerikanisierten Wahlkampfs liegen - in Stichworten - auf: Personalisierung, Symbolisierung, Mediatisierung, Professionalisierung, Entideologisierung, Stimmungssteuerung mit Hilfe von Instrumenten wie Demoskopie, coaching, agenda setting." (Ursula Feist / HansJürgen Hoffmann, Die Bundestagswahl 1998. Wahl des Wechsels, in: ZParl, 30. Jg. (1999), H. 2, S. $215-251$, S. 216).

3 Vgl. Heinz Adamski, System- und Politikverdrossenheit vor der Bundestagswahl, in: Gegenwartskunde, 47. Jg. (1998), H. 3, S. 277 - 283; Kai Arzheimer, Politikverdrossenheit. Bedeutung, Verwendung und empirische Relevanz eines politikwissenschaftlichen Begriffs, Wiesbaden 2002; Christof Erhart / Eberhard Sandschneider, Politikverdrossenheit, in: ZParl, 25. Jg. (1994), H. 3, S. 441 - 453; Martin Greiffenhagen, Politische Legitimität in Deutschland, Gütersloh 1997. 
staatlichen Ebenen ${ }^{4}$ sowie in der schwieriger werdenden Rekrutierung von politisch Aktiven.

Die hier aufgeworfene Frage nach den mittel- bis langfristigen Wahlkampfeffekten auf Wirklichkeitskonstruktionen von Politik ist mit einem großen Dilemma verbunden: Aus wissenschaftlicher Perspektive ist sie von hoher Bedeutung, im Kalkül der Wahlkampfstrategen und politischen Akteure hat sie dagegen bislang kaum Platz gefunden, weil deren Zeithorizont kürzer angelegt ist und vorrangig die Mobilisierung von Wählergunst betrifft. Ebenfalls nur wenig erforscht sind die Wirkungen von Wahlkampf auf noch nicht wahlberechtigte Jugendliche, wenngleich die politischen Einstellungen der 16- und 17-Jährigen in Zusammenhang mit der Diskussion um eine Wahlaltersabsenkung von steigendem Interesse sind. Hier werden beide Fragestellungen miteinander verbunden: Welche Wirklichkeitskonstruktionen von Politik liefert Wahlkampf? Und welche Konsequenzen hat dies auf Einstellungen und Verhalten von noch nicht wahlberechtigten jungen Menschen? Dabei sind zwei Thesen vorstellbar: (1) Wahlkampf wird von den noch nicht wahlberechtigten Jugendlichen gar nicht wahrgenommen, weil deren politisches Interesse zu gering ist. Dann wären auch keine Veränderungen ihrer Einstellungen zu erwarten. (2) Wahlkampf hinterlässt Veränderungen in den Politikperzeptionen Jugendlicher, die sich - so eine weitere Vermutung - auf die Legitimation von Politik negativ auswirken. Sollte letzteres der Fall sein, wäre nach Empfehlungen zu fragen, wie Parteien künftig ihre Wahlkämpfe gestalten sollten.

Die referierten Ergebnisse basieren auf einer standardisierten, anonymen Untersuchung bei 13- bis 18-Jährigen aus Süddeutschland. In einer ersten Untersuchung wurden während des Bundestagswahlkampfs 1998 dieselben Jugendlichen zu drei Zeitpunkten befragt: vor Beginn des Wahlkampfs, unmittelbar vor der Wahl und ein Vierteljahr nach der Wahl. Dabei wurden signifikante Veränderungen der Messwerte durch entsprechende statistische Verfahren erhoben. Zu Beginn der Panel-Untersuchung wurde ein repräsentatives Sample hergestellt. Im weiteren Verlauf kam es aufgrund des Ausfalls von Testpersonen (Krankheit, Schulwechsel) zu Verzerrungen, die aber im Hinblick auf die Fragestellung nicht schwer wiegen. Die Untersuchung wurde während des Bundestagswahlkampfs 2002 im gleichen Setting wiederholt, um spezifische Effekte des jeweiligen Wahlkampfs extrapolieren zu können. Insgesamt nahmen an dieser Untersuchung 5.000 Schüler und Schülerinnen teil. Die geplante dritte Erhebung konnte wegen der plötzlichen Vorverlegung des Wahltermins auf das Jahr 2005 nicht realisiert werden und hätte wegen der gänzlich anderen Struktur des kurzen Wahlkampfs auch keine vergleichbaren Daten geliefert. Ergänzend wurden mit demselben Fragebogen 2.200 Jugendliche in Österreich, der Schweiz und der Russischen Föderation - ebenfalls während nationaler Parlamentswahlen - befragt, um die für Deutschland gefundenen Ergebnisse besser einordnen und Einflüsse der unterschiedlichen Politischen Kulturen extrapolieren zu können. ${ }^{5}$ Das eingesetzte Instrument stellt eine

4 Vgl. Michael Eilfort, Die Nichtwähler: Wahlenthaltung als Form des Wahlverhaltens, Paderborn 1994; Thomas Kleinhenz, Der Nichtwähler. Ursachen der sinkenden Wahlbeteiligung in Deutschland, Opladen 1995; Marion Völker / Bernd Völker, Wahlenthaltung. Normalisierung oder Krisensymptome?, Wiesbaden 1998.

5 Vgl. Michael C. Hermann, Politische Weltbilder in der Mediendemokratie. Eine interdisziplinäre Modellbildung am Beispiel von Wahlkampfkommunikation, Bad Heilbrunn 2009. 
Weiterentwicklung bereits eingeführter und erprobter Skalen zur Erforschung politischer Einstellungen bei Jugendlichen dar. ${ }^{6}$

\section{Jugend und Politik: häufige Missverständnisse}

Junge Menschen in westlichen Demokratien sind politisch desinteressiert, weitgehend inkompetent, kaum zu mobilisieren, apathisch und mit hoch negativen, oft zynischen Einstellungen zum politischen Geschehen ausgestattet - das ist die landläufige öffentliche Meinung zum Verhältnis der jungen Generation zur Politik. Eine Auswertung empirischer Studien zeigt deutlich, dass dies Klischees sind, die der Wirklichkeit kaum gerecht werden. Hinzu kommen eine Reihe von Missverständnissen und Fehlinterpretationen.

Als politische Einstellungen (attitudes) werden im wissenschaftlichen Diskurs das politische Interesse, die zugeschriebene politische Kompetenz, die politische Wirksamkeit, das prospektive politische Engagement, die individuelle Durchschaubarkeit politischer Prozesse und der politische Zynismus untersucht. Sie machen einen großen Teil der Politischen Kultur, also der „Gesamtheit aller politisch relevanten individuellen Persönlichkeitsmerkmale, latente, in Einstellungen und Werte verankerten Prädispositionen zu politischem Handeln - auch in ihren symbolhaften Ausprägungen - und hieraus abgeleitetes politisches Verhalten "7 aus. Zu allen eben genannten Variablen existieren gut eingeführte und erprobte Skalen.

\section{Politisches Interesse}

Traditionell wird diese Variable operationalisiert mit: „Ich interessiere mich für Politik“. Die Abfrage des so genannten Single-Items zeigt ein bei Jugendlichen nur geringes politisches Interesse, das bei männlichen, älteren Befragten und solchen mit einem höheren formalen Bildungsniveau besser ausfällt. Diese Operationalisierung spiegelt ein erstes Missverständnis und wird der Realität kaum gerecht. ${ }^{8}$ Festzustellen ist eine zunehmende Spaltung der kognitiven Mobilisierung Jugendlicher erstens in ein auf das Geschehen auf der politi-

6 Vgl. ders., Jugendgemeinderäte in Baden-Württemberg. Eine interdisziplinäre Evaluation, Pfaffenweiler 1996; Hans-Peter Kuhn, Mediennutzung und politische Sozialisation. Eine empirische Studie zum Zusammenhang zwischen Mediennutzung und politischer Identitätsbildung bei Jugendlichen, Opladen 2000; Ursula Hoffmann-Lange / Dagmar Krebs / Johann de Rijke, Kognitive politische Mobilisierung und politisches Vertrauen, in: Ursula Hoffmann-Lange (Hrsg.), Jugend und Demokratie in Deutschland. DJI-Jugendsurvey 1, Opladen 1995, S. 359 - 387; Angelika Vetter, Political Efficacy - Reliabilität und Validität. Alte und neue Massenmodelle im Vergleich, Wiesbaden 1997; Oscar Gabriel, Politische Einstellungen und politisches Verhalten, aus: ders. I Everhard Holtmann (Hrsg.), Politisches System der Bundesrepublik Deutschland, München / Wien 1999, S. 381 - 497, S. 431 ff.; Martina Gille / Winfried Krüger (Hrsg.), Unzufriedene Demokraten. Politische Orientierungen der 16- bis 29-Jährigen im vereinigten Deutschland. DJIJugendsurvey 2, Opladen 2000; Rainer Watermann, Politische Sozialisation von Kindern und Jugendlichen, in: APuZ, B 41 (2005), S. 16 - 24.

7 Dirk Berg-Schlosser, Erforschung der Politischen Kultur - Begriffe, Kontroversen, Forschungsstand, in: Gotthard Breit (Hrsg.), Politische Kultur in Deutschland. Abkehr von der Vergangenheit - Hinwendung zur Demokratie, 36. Jg. (2003), H. 3, S. 7 - 20, S. 7.

8 Vgl. Ursula Hoffmann-Lange / Dagmar Krebs / Johann de Rijke, a.a.O. (Fn. 6). 
schen Bühne gerichtetes Interesse und zweitens in ein Interesse an der Lösung drängender gesellschaftlicher Probleme. Die erste Dimension kann als konventionelle politische Mobilisierung bezeichnet werden, die zweite als alternative oder objektbezogene. Die konventionelle politische Mobilisierung wird gut durch das oben vorgestellte Single-Item erfasst, die alternative kann durch Formulierungen wie „Ich interessiere mich dafür, wie unsere Gesellschaft Probleme wie (...) löst" operationalisiert werden. Diese alternative Mobilisierung liegt in allen befragten Gruppen wesentlich höher als die konventionelle und weist auch nicht die strukturellen Unterschiede nach Geschlecht, Alter und Bildungsniveau auf. Es markiert das Niveau der Mobilisierbarkeit der jungen Generation.

Die Aussage, Jugendliche seien politisch desinteressiert, ist also in dieser Vereinfachung nicht zutreffend. Vielmehr gilt, dass das Interesse Jugendlicher am Geschehen auf der politischen Bühne gering und wohl auch rückläufig ist, während doch erhebliche Betroffenheit und Aktivation gegenüber drängenden politisch-gesellschaftlichen Problemen und deren Lösung bestehen. Diese Spaltung des politischen Interesses ist problematisch und offenbart die kritische Distanz, die sich bei Jugendlichen gegenüber den Routinen des politisch-administrativen Systems eingestellt hat. Weiter unten wird zu fragen sein, wie das konventionelle und alternative politische Interesse Jugendlicher auf Wahlkampf reagieren.

\section{Politische Kompetenz}

Ein zweites wichtiges Einstellungsfeld ist die zugeschriebene politische Kompetenz. Es wird hinsichtlich der Objekte Parteien, Behörden und Politiker meist differenziert erhoben. In der hier vorliegenden Untersuchung wurde die zugeschriebene politische Kompetenz operationalisiert mit „Ich finde, dass ... gute Arbeit leisten“. Bei den deutschen Befragten erreichen Parteien, Politiker und Behörden etwa gleiche Werte. Eindeutig gute Arbeit bescheinigen nur drei Prozent der deutschen Jugendlichen den verschiedenen Akteuren, eher gute Arbeit ein Drittel. Im Vergleich zu den anderen untersuchten Ländern wird nur in der Schweiz höhere Zustimmung erzielt. In Russland schneiden erwartungsgemäß die Behörden besonders schlecht $\mathrm{ab}$.

\section{Politische Wirksamkeit}

Politische Wirksamkeit ist als die individuelle Auffassung zu verstehen, dass das politische System die Interessen des Einzelnen ernst nimmt und dass dieser durch eigenes Handeln Einfluss entfalten kann auf den Verlauf des politischen Prozesses und dessen Ergebnisse. Politische Wirksamkeit wird entsprechend der englischsprachigen Literatur differenziert in „internal efficacy“ und „external efficacy“. Erstere hebt auf die eigenen Kompetenzen (Ausdrucksfähigkeit, Sachverstand) ab und wird meist operationalisiert durch: „Ich denke, dass auch ich wichtige Meinungen und Vorschläge zu politischen Fragen beisteuern könnte“. „External efficacy“ bezeichnet den Glauben an die Responsivität des politischen Systems und wird regelmäßig negativ umgesetzt: „Politiker kümmern sich nicht darum, was junge Menschen wollen“ oder „Was Politiker machen, geschieht unabhängig davon, was die Menschen wollen." Im aktuellen Diskurs wird insbesondere das Einstellungsfeld Wirksamkeit als sehr relevant für die Mobilisierung von Legitimation gegenüber dem politischen System gesehen. In einer Reihe von Studien wurde im Rahmen von Varianzanalysen gezeigt, dass empfundene Wirksamkeit positive Effekte auf die Ausprägung anderer politischer Einstel- 
lungen hat. ${ }^{9}$ Nur etwa die Hälfte der befragten deutschen Jugendlichen bestätigt „,external political efficacy“, nur ein Drittel „internal efficacy“, wobei hier kaum Unterschiede nach Alter, Geschlecht und Bildungsniveau ersichtlich sind. Auch die Unterschiede zwischen Deutschland, Österreich, der Schweiz und der Russischen Föderation sind gering.

\section{Durchschaubarkeit}

Der Satz „Die ganze Politik ist so kompliziert, dass ich oft nicht verstehe, was wirklich vor sich geht" operationalisiert das Einstellungsfeld der individuellen Durchschaubarkeit. Etwas weniger als die Hälfte der Befragten sind eindeutig oder eher der Auffassung, nicht verstehen zu können, was auf der politischen Bühne geschieht. Dieses Gefühl ist bei den weiblichen Befragten besonders stark ausgeprägt. Aus der Literatur ist dieses Phänomen bekannt. Es wird darauf zurückgeführt, dass Frauen mehr an einer konstruktiven Auseinandersetzung mit Sachfragen und weniger am auf der Bühne inszenierten Streit interessiert sind. Oder in anderen Worten: Für sie ist die policy-Perspektive attraktiver als die politics-Ebene.

\section{Politischer Zynismus}

Mit politischem Zynismus ist der tief verwurzelte Argwohn hinsichtlich der Motive und Handlungen politischer Akteure gemeint. Er ist in dem hier untersuchten Sample weniger stark ausgeprägt als erwartet. Etwa ein Viertel der befragten deutschen Jugendlichen meint, dass „Politik ein schmutziges Geschäft“ sei. Etwas mehr stimmen dem Item „Die meisten Politiker sind arrogant und unfähig" zu. Kaum abgelehnt wird die Sinnhaftigkeit von Wahlen und Parteien. Jugendliche in der Schweiz zeigen weniger Zynismus, österreichische dagegen mehr, was den so genannten österreichischen Theaterdonner, einen Mechanismus bewusster Konfliktkommunikation zur Verschleierung von Konkordanzbemühungen, illustriert.

\section{Systemvertrauen}

Unter Systemvertrauen wird die rational wie emotional grundierte Einstellung verstanden, dass die pluralistische, repräsentative, rechtstaatliche und freie Parteiendemokratie in ihrer konkreten Ausprägung positiv zu bewertende Leistungen für die Individuen erbringt. Es kann als eine Bilanz der oben genannten Einstellungsfelder verstanden werden. Systemvertrauen in diesem Sinne ist eng verwandt mit dem von David Easton ${ }^{10}$ entwickelten Konzept des „specific“ und „diffuse support“. Während ersteres als Zustimmung verstanden wird, dass die Politikergebnisse den Bedürfnissen des Individuums gerecht werden, bedeutet letztere die Zustimmung zu den Ordnungsprinzipien an sich. Gert Pickl und Dieter Walz haben allerdings darauf hingewiesen, dass diese analytische Trennung problematisch ist. In ihren Untersuchungen ergaben sich „doch erstaunlich starke Einflüsse der Regie-

9 Vgl. Gert Pickel / Dieter Walz, Politikverdrossenheit in Ost- und Westdeutschland: Dimensionen und Ausprägungen, in: PVS, 38. Jg. (1997), H. 1, S. 27 - 49, S. 47; Gert Pickel, Jugend und Politikverdrossenheit. Zwei politische Kulturen in Deutschland nach der Vereinigung?, Opladen 2002.

10 Vgl. David Easton, A System Analysis of Political Life, New York 1965; Dirk Berg-Schlosser, a.a.O. (Fn. 7), S. 11. 
rungsbewertung auch auf die theoretisch eigentlich nahezu unabhängigen diffusen Komponenten des politischen Systems“. Dies kann so interpretiert werden, „dass eine dauerhafte Unzufriedenheit mit den politischen Vertretern und deren Effektivität auf lange Sicht auch negative Effekte auf die Systemstabilität als Ganze besitzen kann"11.

In der Literatur findet sich eine Reihe unterschiedlicher Operationalisierungen zu Systemvertrauen beziehungsweise Systemunterstützung. ${ }^{12}$ In der vorliegenden Untersuchung wurde die Systemunterstützung abgefragt durch das Item „Politik und Staat funktionieren gut und brauchen keine wesentliche Veränderung“. Diese, von anderen Untersuchungen abweichende Operationalisierung wurde gewählt, um eine ausreichende kognitive Präsenz bei den befragten Jugendlichen - darunter auch 13-Jährige - sicherzustellen. Operationalisierungen, die zentral auf das Demokratieprinzip und seine Umsetzung abzielen, hätten das Problem aufgeworfen, dass Artefakte aufgrund unzureichender kognitiver Präsenz nicht hätten ganz ausgeschlossen werden können. Innerhalb des Untersuchungszeitraums schwankte der Anteil der deutschen Jugendlichen, die dieser Aussage ganz oder eher zustimmten, zwischen 15 und 25 Prozent. Die Ergebnisse präsentieren sich als weitgehend unabhängig von Alter, Geschlecht und besuchter Schulform. Dieser Befund illustriert, wie dünn die Legitimation des politisch-administrativen Systems bei der nachwachsenden Generation und wie drängend eine Auseinandersetzung mit diesem Phänomen geworden ist. Dabei sollte nicht vergessen werden, dass derart grundsätzliche Attitüden - wie verschiedene balancetheoretische Ansätze ${ }^{13}$ beschreiben - von hoher Stabilität sind. Daher muss erwartet werden, dass sich die hier untersuchte Alterskohorte auch später in kritischer Distanz zur Politik befinden wird.

\section{Wie Wahlkampf auf Jugendliche wirkt: Überlegungen zur Modellbildung}

Die Annahme, dass der von Parteien organisierte Wahlkampf direkt auf die Einstellungsebene von Menschen wirke, ist eine unzulässige Vereinfachung. Zunächst ist Wahlkampf eine

11 Gert Pickl/ Dieter Walz, Politikverdrossenheit in Ost- und Westdeutschland: Dimensionen und Ausprägungen, in: PVS, 38. Jg. (1997), H. 1, S. 27 - 49, S. 42 f.

12 Dieter Fuchs und Edeltraut Roller beispielsweise wählen für die mehr spezifische Systemunterstützung eine Operationalisierung anhand der drei Ebenen Einstellungen zur Demokratie an sich, Einstellung zu der in Deutschland institutionalisierten Demokratie und Einstellung zur Demokratie, wie sie in Deutschland faktisch funktioniert. (Dieter Fuchs / Edeltrand Roller, Die Einstellung zur Demokratie in Deutschland, in: Gotthard Breit (Hrsg.), a.a.O. (Fn. 7), S. 21 - 26, S. 21.) Die Unterstützung demokratischer Prinzipien wird von Oscar W. Gabriel operationalisiert als additiver Index aus der Zustimmung zum Recht, für seine Meinung einzutreten, und der Zustimmung zur Feststellung, dass Demokratie ohne Opposition nicht denkbar ist. (Oscar W. Gabriel, Aktivisten als Träger des demokratischen Credos? Zum Zusammenhang zwischen politischer Partizipation und der Unterstützung demokratischer Prinzipien im vereinten Deutschland, in: Oskar Niedermayer / Bettina Westle (Hrsg.), Partizipation und Demokratie. Festschrift für Max Kaase, Wiesbaden / Opladen 2000, S. 34 - 45, S. 39). Jan W. van Deth operationalisiert die Zufriedenheit mit Politik durch die Zufriedenheit mit dem Funktionieren des politischen Systems, der Zufriedenheit mit der Demokratie, mit der Regierung, der Wirtschlage Deutschlands und der Wirtschaftslage der Befragten selbst. (Jan W. van Deth, Das Leben, nicht die Politik ist wichtig, in: Oskar Niedermayer / Bettina Westle, a.a.O. (Fn. 12), S. 115 - 135, S. 128 f.)

13 So zum Beispiel Leon Festingers Theorie der kognitiven Dissonanz. Vgl. Leon Festinger, A Theory of Cognitive Dissonance, Stanfort 1962. 
von politischen Akteuren verantwortete Kommunikationsleistung, die in der Erwartung von Wirksamkeit im publizistischen System und bei den Rezipienten organisiert wird. Die „spin doctors“, Kampa-Manager und Wahlkampfstrategen vertrauen dabei auf eine Reihe von Feedback-Schleifen wie zum Beispiel der Demoskopie, um die Effizienz ihrer kommunikativen Bemühungen sicherzustellen. Unzulässig vereinfacht wäre auch die Vorstellung, dass die politischen Akteure Macht über das publizistische System hätten, letzteres also den Input der Politik einfach an den Rezipienten weiter transportieren würde. Die Kommunikationsexperten der Parteien analysieren tatsächlich die Selektions- und Präsentationsprinzipien der Massenmedien und stellen sich auf diese ein. Insofern zwingt das massenmediale System seine Bedingungen dem politischen System auf. Die im wissenschaftlichen Diskurs immer wieder diskutierte Frage, wer denn nun mehr Macht habe - Politik oder Publizistik -, hat damit keine große Bedeutung. ${ }^{14}$ Beide Systeme wirken gleichzeitig aufeinander. Einmal setzen sich eher die Codes des publizistischen, ein anderes Mal eher jene des politischen Systems durch. Massenmedien verstärken also nicht etwa den Input der Wahlkampfmanager, sie selektieren ihn und präsentieren diesen auf die ihnen eigene Art und Weise. Mitunter durchkreuzen sie die Kommunikationsstrategien der Parteien.

Für die hier zu klärende Frage, wie sich Wahlkampfkommunikation auf jugendliche Rezipienten auswirkt, ist es notwendig, neben den Strategien der Parteien vor allem auch die Medialisierungsleistungen der Massenmedien in den Blick zu nehmen. Die vom Journalismus vorgenommene Selektion lässt sich nach dem derzeitigen Stand des medienwissenschaftlichen Diskurses am besten mit Hilfe der Nachrichtenwerttheorie beschreiben. ${ }^{15}$ Diese vom norwegischen Friedensforscher Johan Galtung ${ }^{16}$ geprägte Theorie geht auf wahrnehmungspsychologischer Basis von folgender Grundannahme aus: Journalisten wie Rezipienten weisen ihre Aufmerksamkeit je nach Stärke von in einer Nachricht vermuteten Faktoren zu. Als solche Nachrichtenfaktoren wurden vor allem Eindeutigkeit, Negativität, Personalisierung, Überraschung und Aktualität extrapoliert. Je mehr dieser Faktoren und je stärker sie in einer Nachricht vorhanden sind, desto höher ist der zugemessene Nachrichtenwert und damit die Aufmerksamkeit. Ferner kann gesagt werden: Je jünger ein Rezipient ist, je weniger wirksam die mit der Lebenszeit erworbenen Schemata und Heuristiken sind, desto stärker wird der Einfluss der Nachrichtenfaktoren in der Rezeption sein. Zusätzlich zu der Selektion wird auf jeder Ebene des massenmedialen und informellen Kommunikationsprozesses eine Betonung derjenigen Teile der Nachricht vorgenommen, die mit starken Faktoren verbunden sind. Elemente der Nachricht, die keinen hohen Nachrichtenwert haben, werden ausgeblendet. So findet eine Verzerrung von Realität statt. Über diese Mechanismen sind sich die Wahlkampfstrategen und Kommunikationsprofis im Klaren, so dass sie Ereignisse, Themen und Nachrichten in einer Weise inszenieren, dass ihnen ein hoher Nachrichtenwert zukommt. So lässt sich auch die Bedeutung von „negative campaigning“

14 Vgl. zu diesem Diskurs Yong-Yoo Choi, Interpenetrationen von Politik und Massenmedien, Münster / Hamburg 1995.

15 Vgl. grundlegend Winfried Schulz, Die Konstruktion von Realität in den Nachrichtenmedien. Analyse der aktuellen Berichterstattung, Freiburg / München 1990.

16 Vgl. Johan Galtung / Mari Holmboe Ruge, The Structure of Foreign News. The Presentation of the Congo, Cuba, Cyprus Crisis in Four Norwegian Newspapers, in: Journal of Peace Research, 2. Jg. (1965), H. 1, S. $65-91$. 
und von Personalisierung theoretisch erklären. ${ }^{17}$ „Negative campaigning“" nutzt die wahrnehmungspsychologisch abgesicherte Attraktivität des Negativen aus. Personalisierung dient der Komplexitätsreduktion bei beständig steigender politischer Komplexität. Dies liefert deutliche Hinweise darauf, dass sich die bei Jugendlichen unter Wahlkampfbedingungen einstellende Wirklichkeitskonstruktion insbesondere durch Negativität, Personalisierung und Vereinfachung auszeichnen dürfte.

Eine große theoretische wie auch empirische Herausforderung stellt die Frage dar, wie sich die massenmedial generierte politische Wirklichkeit auf das kognitive System der Rezipienten auswirkt. Eindeutig widerlegt ist eine Vorstellung, wonach das Bewusstsein der Rezipienten das massenmedial Generierte als Diapositiv widerspiegelt, es also eine direkte Stimulation von Einstellungen, Wissen und Verhalten durch den publizistischen Input gibt. ${ }^{18}$

Seit Beginn der modernen Medienwirkungsforschung ${ }^{19}$ war die Beeinflussung politischer Einstellungen den Wissenschaftlern große Aufmerksamkeit wert. Heutige Auffassung ist, dass ältere Ansätze ${ }^{20}$ als einzelne nicht geeignet sind, die komplexe Informationsaufnahme und -verarbeitung durch Rezipienten zu beschreiben. Entwickelt wurden deshalb integrative und dynamische Modelle, in denen diese unterschiedlichen Theorien und Ansätze gleichermaßen ihren Platz finden.

Ein solcher mit dem dynamisch-transaktionalen Modell der Medienwirkungsforschung vorliegender Zugriff erlaubt, Medienwirkungen im Hinblick auf bestimmte Rezipientengruppen in bestimmten sozialen Situationen genauer zu beschreiben. ${ }^{21}$ Als Modell erklärt der in Deutschland wesentlich von Werner Früh geförderte Ansatz keine konkreten Wirkungen. Er stellt eine Grundlage dar für die Konzeption empirischer Untersuchungen, wobei hochkomplexe Settings einerseits und die Verabschiedung von einfachen Ursache-Wirkungszusammenhängen andererseits die Konsequenz sind. Zentral in diesem Modell ist,

17 Vgl. Hans Mathias Kepplinger / Rouwen Bastian, Der prognostische Gehalt der NachrichtenwertTheorie, in: Publizistik, 45. Jg. (2000), H. 4, S. 462 - 475; Werner Früh, Realitätsvermittlung durch Massenmedien. Die permanente Transformation der Wirklichkeit, Opladen 1994; Christiane Eilders, Nachrichtenfaktoren und Rezeption. Eine empirische Analyse zur Auswahl und Verarbeitung politischer Information, Opladen 1997.

18 Vgl. Ernst Pöppel (Hrsg.), Grenzen des Bewusstseins. Wie kommen wir zur Zeit, und wie entsteht Wirklichkeit, Frankfurt am Main 1997.

$19 \mathrm{Vgl}$. Heinz Bonfadelli, Medienwirkungsforschung I: Grundlagen und theoretische Perspektiven, Konstanz 1999; ders., Medienwirkungsforschung II. Anwendungen in Politik, Wirtschaft und Kultur, Konstanz 2000.

20 Etwa der der Reiz-Reaktions-Ansatz: vgl. Bernard R. Berelson / Paul F. Lazarsfeld / William N. McPhee, Voting. A Study of Opinion Formation in a Presidential Campaign, Chicago / London 1954. Der Nutzenansatz: vgl. Jay G. Blumler (Hrsg.), The Uses of Mass Communication: Current Perspectives on Gratifications Research, Beverly Hills 1974. Der Thematisierungsansatz: vgl. Maxwell McCombs, Setting the Agenda: The News Media and Public Opinion, Cambridge 2004; Frank Brettschneider, Die Medienwahl 2002: Themenmanagement und Berichterstattung, in: APuZ, B 49/50 (2002), S. 36 - 47; Patrick Rössler, Agenda-Setting. Theoretische Annahmen und empirische Evidenzen einer Medienwirkungshypothese, Opladen 1997. Der Two- beziehungsweise Multi-Step-Flow of Communication Approach: vgl. Cornelia Eisenstein, Meinungsbildung in der Mediengesellschaft. Eine theoretische und empirische Analyse zum Multi-Step-Flow of Communication, Opladen 1994; vgl. Elihu Katz / Paul F. Lazarsfeld, Personal Influence. The Part Played by People in the Flow of Mass Communication, Glencoe 1955.

21 Vgl. Werner Früh (Hrsg.), Medienwirkungen. Das dynamisch-transaktionale Modell, Opladen 1991. 
dass die frühere Vorstellung von Ursache und Wirkung, die Trennung in unabhängige und abhängige Variable überwunden ist. Das Bild der Stimulation wird abgelöst durch Transaktionen. Transaktion ist gegeben, wenn ein Faktor A, der Faktor B beeinflusst, sich durch diese Beeinflussung selbst verändert. Ferner gilt, dass der beeinflusste Faktor B den Faktor A beeinflusst und sich darunter wiederum selbst verändert. „Bei Transaktionen könnte man anstatt von einer einseitigen (...) von einer doppelseitigen (nicht wechselseitigen!) Kausalität sprechen." 22 Die Vertreter dieses Ansatzes sehen eine ganze Reihe verschiedener transaktionaler Verhältnisse bei der Rezeption von Information.

Die Prozesse zwischen Rezipient und medialer Botschaft werden Inter-Transaktionen genannt. Sie „sind imaginäre und reale Interaktionsprozesse zwischen Kommunikatoren und Rezipienten. (...) Beide am Kommunikationsprozess beteiligten Parteien versuchen, in der Inter-Transaktion bewusst oder unbewusst, ihre jeweiligen Gratifikationen aus der Kommunikation zu maximieren" 23 . Die mediale Botschaft wird erst zu einer Botschaft, indem sie einen Rezipienten trifft und von diesem perzipiert wird. Dies richtet den Blick der Medienwirkungsforschung weg von der isolierten Betrachtung einer Wahlkampfbotschaft, die angeblich die identische Anregung für jeden Rezipienten transportiert, hin auf die große Variation unterschiedlicher Rezipienten mit sehr verschiedenen Persönlichkeitseigenschaften, eingesetzten Stereotypen, Schemata und Heuristiken.

Auf die hier diskutierte Fragestellung bezogen ergibt sich damit folgendes Grundmodell: Wirkung von Wahlkampfkommunikation auf Jugendliche ist zu verstehen als das Resultat von Inter-Transaktionen zwischen einer vom Kommunikator intentional gemachten Botschaft, die aber ohne eindeutige Identität bleibt, und den jugendlichen Rezipienten mit deren Prädispositionen. Diese Prädispositionen wiederum sind als Resultat komplexer Intra-Transaktionen im kognitiven System zu verstehen, bei denen die Zuwendung zur Botschaft (Aktivation), das Vorwissen und das habitualisierte Nutzungsverhalten wesentliche Elemente sind. Das dynamisch-transaktionale Modell berücksichtigt auch die Anschlusskommunikation in sozialen Netzwerken, wodurch im Rahmen von Intra-Transaktionen weitere Perzeptionen mitbestimmt werden. All dies versucht das Dynamisch-transaktionale Modell abzubilden. „Die Dimension der Zeit, die Abfolge kommunikativer Prozesse ist integraler Bestandteil des Modells. Dadurch werden nicht nur quantitative, sondern auch qualitative Veränderungen im Wirkungsprozess sichtbar. " 24 Die Vertreter des Modells fordern deshalb Longitudinalstudien sowie experimentelle Settings, wie sie beispielsweise in dem hier referierten Forschungsprojekt realisiert sind.

\section{Rezeption politischer Informationen durch Jugendliche}

Hinsichtlich der Rezeption politischer Informationen durch Jugendliche ist die Datenlage weit weniger gut als für Erwachsene. Insbesondere zur Relevanz des Internets gibt es kaum verlässliche Erkenntnisse. Unstrittig ist aber, dass innerhalb der Mediennutzung Jugendlicher die Aufnahme politischer Informationen eine nur geringe Rolle spielt. Gesichert ist 
ferner der Zusammenhang von Informationsnutzung einerseits und wichtigen Persönlichkeitsvariablen andererseits. „Neben dem Alter ist die Bildung der wichtigste Einflussfaktor für das Informationsinteresse. Mit ansteigender formaler Bildung nimmt auch das Interesse an ,aktuellen Ereignissen' zu. "25

In der hier referierten Untersuchung stimmten, mit Schwankungen je nach Untersuchungstranche, durchschnittlich 21 Prozent der befragten Jugendlichen dem Statement: „Ich lese / höre / sehe häufig Nachrichten in Zeitung, Radio oder TV, die sich mit Politik beschäftigen" voll zu. Weitere rund 36 Prozent stimmten eher zu. Der Mittelwert auf einer ansteigenden fünfteiligen Skala liegt bei 3,5. Jungen erreichen im Durchschnitt 0,5 Punkte höhere Werte. Die Mittelwerte steigen mit dem Alter wie erwartet leicht an, ebenso mit dem Bildungsniveau (vgl. Tabelle 1).

Die Tageszeitung hat als Medium der politischen Information in der Wertschätzung Jugendlicher in den letzten Jahren überraschenderweise zugenommen. Diese abstrakte $\mathrm{Zu}-$ stimmung geht allerdings einher mit einer stark rückläufigen Reichweite und einer stark selektiven Nutzung.

Hinsichtlich des Fernsehens kommen alle Studien zum Ergebnis, dass die Rezeption politischer Informationen aus diesem Medium bei Jugendlichen nur eine geringe quantitative Rolle spielt. Politische Magazine gelten als besonders unattraktiv. „Politikvermittlung in den klassischen Magazinen erfolgt sach-, weniger personenbezogen, stärker abstrakt als konkret und personalisiert, häufiger unter dem gesamtgesellschaftlichen Blickwinkel als boulevardesk in Form von Infotainment aufbereitet. Gerade diese formal-inhaltlichen Kriterien bedingen das Urteil vieler Jugendlicher, dass Informationssendungen langweilig und an den Interessen Jugendlicher vorbei produziert sind. Dagegen scheint Personalisierung und Nachvollziehbarkeit der Schlüssel zur Aufmerksamkeit der Jugendlichen zu sein."26 Hans-Peter Kuhn vertritt die Auffassung, dass Nachrichtensendungen im Fernsehen bei Jugendlichen „eine chaotische Ansammlung von Fakten“ fördern, es werde „immer mehr gewusst und immer weniger verstanden. (...) Die psychische Überforderung kann bei Kindern und Jugendlichen dann vielfältige politische Ängste auslösen "27. Unbeschadet der geringen Nutzung des Fernsehens als Quelle politischer Information wird diesem Medium von den Jugendlichen hohe Relevanz und Glaubwürdigkeit bescheinigt. Insbesondere dem öffentlich-rechtlichen Rundfunk wird „hohe Informationskompetenz zuerkannt, trotz der rückläufigen Nutzung dieser Sparte “28. Empirische Forschung ergab ferner, dass individuell negative politische Einstellungen gut erklären können, warum Informationsangebote des öffentlich-rechtlichen Rundfunksystems wenig wahrgenommen werden. „So könnte die zutage getretene deutliche Skepsis der Jugendlichen gegenüber tradierten gesellschaftlichen

25 Sascha Blödorn / Maria Gerhards / Walter Klingler, Informationsnutzung und Medienauswahl. Ergebnisse einer Repräsentativbefragung zum Informationsverhalten der Deutschen, in: Media Perspektiven, 35. Jg. (2005), H. 12, S. 638 - 646, S. 639.

26 Birgit van Eimeren / Brigitte Maier-Lesch, Mediennutzung und Freizeitgestaltung von Jugendlichen. Ergebnisse einer Repräsentativbefragung von rund 1000 Jugendlichen zwischen zwölf und 19 Jahren, in: Media Perspektiven, 27. Jg. (1997), H. 11, S. 590 - 603, S. 600.

27 Hans-Peter Kuhn, a.a.O. (Fn. 6), S. 68.

28 Marlene Wösle, Öffentlich-rechtliches Fernsehen: Für Jugendliche nicht jung genug? Nutzungsverhalten und Präferenzen junger Leute beim Fernsehen, in: Media Perspektiven, 29. Jg. (1999), H. 11, S. $583-590$, S. 584. 


\begin{tabular}{|c|c|c|c|c|c|c|}
\hline & \multirow{6}{*}{ 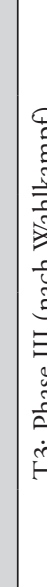 } & 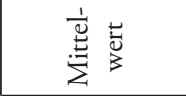 & $\begin{array}{l}6 \\
\dot{m}^{\prime}\end{array}$ & $\begin{array}{ll}\infty & \infty \\
\dot{m} & \infty\end{array}$ & $\begin{array}{ll}* & r \\
m & \pi\end{array}$ & \multirow{19}{*}{ 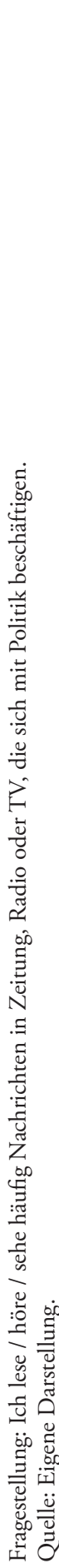 } \\
\hline & & 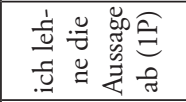 & $\begin{array}{cc}0 & 0 \\
m & i n\end{array}$ & $\begin{array}{c}0 \\
i \\
i\end{array}$ & $\hat{m} \vec{n}$ & \\
\hline & & 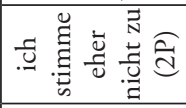 & $\begin{array}{l}\hat{\Xi} \\
\hat{\beth}\end{array}$ & $\begin{array}{l}\infty \\
\stackrel{ \pm}{ \pm}\end{array}$ & 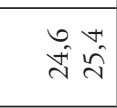 & \\
\hline & & 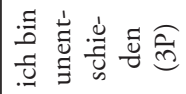 & 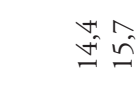 & $\overrightarrow{\mathfrak{c}} \stackrel{?}{\mathfrak{c}}$ & $\hat{\sigma}=$ & \\
\hline & & 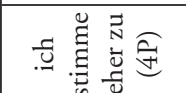 & \begin{tabular}{l}
$\forall+t$ \\
\multirow{2}{*}{$\hat{m}$}
\end{tabular} & 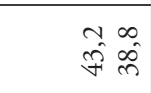 & $\begin{array}{ll}\hat{\sigma} & 0 \\
\hat{F} & 0 \\
\hat{n}\end{array}$ & \\
\hline & & 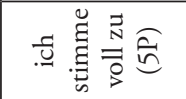 & $\stackrel{n}{=} \approx$ & 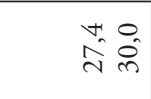 & $\begin{array}{l}\approx n \\
\Rightarrow \approx\end{array}$ & \\
\hline \multirow{6}{*}{\multicolumn{2}{|c|}{ 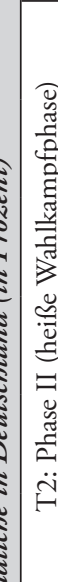 }} & 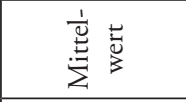 & $\begin{array}{l}* \\
m \\
m\end{array}$ & $\dot{m} \hat{m}$ & $\stackrel{m}{m}$ के & \\
\hline & & 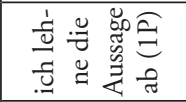 & $\begin{array}{l}m_{0}^{\infty} \\
\forall i n\end{array}$ & 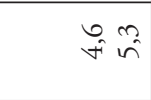 & 우 & \\
\hline & & 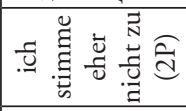 & $\begin{array}{l}00 \\
\hat{i} \\
\vec{\lambda}\end{array}$ & 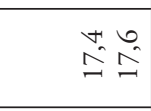 & $\begin{array}{ll}0 & 0 \\
0 & 0 \\
i & \ddots\end{array}$ & \\
\hline & & 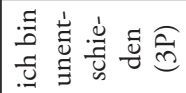 & $\begin{array}{l}\hat{\theta} \\
\hat{n}\end{array}$ & $\begin{array}{ll}\hat{0} & 0 \\
\hat{\sigma} & \stackrel{-}{1}\end{array}$ & $\begin{array}{l}\forall+\infty \\
\stackrel{-}{=}\end{array}$ & \\
\hline & & 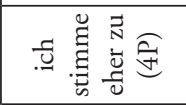 & 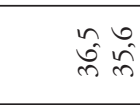 & 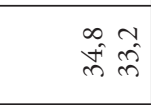 & $\begin{array}{ll}m & 0 \\
\infty & 0 \\
n & \infty\end{array}$ & \\
\hline కัँ & & 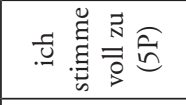 & 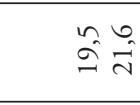 & તુ & ิิ & \\
\hline$\frac{5}{5}$ & \multirow{6}{*}{ (2) } & 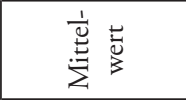 & $\begin{array}{c}\pi \\
m \\
m\end{array}$ & $\tilde{m} \tilde{m}$ & $\begin{array}{c}0 \\
\text { mi m }\end{array}$ & \\
\hline 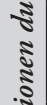 & & 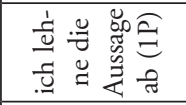 & $\hat{\sigma} \sigma$ & $\begin{array}{cc}m & 0 \\
0 & \sigma\end{array}$ & $\stackrel{n}{\sim} \stackrel{*}{\sigma}$ & \\
\hline$\frac{5}{5}$ & & 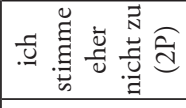 & $\begin{array}{l}\hat{\hat{v}} \\
\hat{d}\end{array}$ & $\begin{array}{l}\approx \approx \\
\hat{\imath}\end{array}$ & $\begin{array}{l}m 0 \\
\stackrel{m}{m}\end{array}$ & \\
\hline 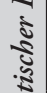 & & 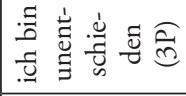 & $\hat{\imath}$ & $\begin{array}{ll}0 & \infty \\
\mathbb{J}^{\hat{U}} & \hat{f}\end{array}$ & $\vec{\approx}$ & \\
\hline 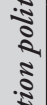 & & 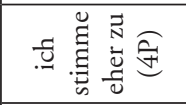 & $\begin{array}{l}0 \text { v } \\
\text { nี } \tilde{n}\end{array}$ & 辛 & $\begin{array}{l}\hat{m} \\
\dot{n}\end{array}$ & \\
\hline ๕ूँ & & 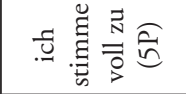 & $\stackrel{\infty}{0}$ & $\hat{\tilde{\imath}} \hat{\tilde{\lambda}}$ & $\hat{n}=$ & \\
\hline 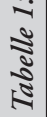 & & & 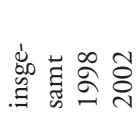 & 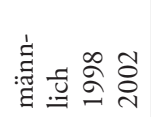 & 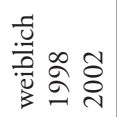 & \\
\hline
\end{tabular}


Institutionen zumindest teilweise das schlechte Image des öffentlich-rechtlichen Fernsehens und die Abkehr der Jugendlichen von diesen Sendern erklären. “29

Der Hörfunk hat in den letzten beiden Jahrzehnten seine Bedeutung als Quelle politischer Information bei Jugendlichen weitgehend verloren. Noch 1990 hatte er „die höchste Chance, Jugendliche mit politischen Inhalten zu erreichen. Erst dann folgte das Fernsehen, schließlich abgeschlagen die Tageszeitung "30. Nach der Durchformatierung der Programme und einer deutlichen Reduktion des Wortanteils werden die Sender mit moderner Musikfarbe von Jugendlichen fast nur noch als so genanntes Nebenbei-Medium genutzt (vgl. Tabelle 2).

Die im vorliegenden Forschungsprojekt gefundenen Strukturen der Mediennutzung entsprechen denen aus dem wissenschaftlichen Diskurs bekannten. Zeitungsnutzer sind tendenziell etwas ältere Jugendliche und häufiger Jungen als Mädchen. Unter den Radiohörern finden sich stark überdurchschnittlich Mädchen, unter den Fernsehzuschauern mehr Jungen. Tendenziell nimmt die Nutzung der Tageszeitung während des Untersuchungszeitraums, vor allem während der heißen Wahlkampfphase, zu.

Jugendliche mit einer ausgeprägten Affinität zu Tageszeitungen wiesen eine signifikant stärkere Mobilisierung gegenüber Politik auf, wobei dieses Interesse während des Wahlkampfs nochmals anstieg. Auch zeigte sich bei ihnen eine stärker empfundene Durchschaubarkeit von Politik. Diese Gruppe Jugendlicher unterscheidet sich von den Gleichaltrigen auch dadurch, dass sie Politik weniger stark personalisiert und negativ erlebt. Ferner ist bei ihnen die externe und interne Wirksamkeit stärker, der politische Zynismus dagegen schwächer ausgeprägt. Die Daten offenbaren deutliche Interaktionen der individuellen Wahrnehmung einer stark personalisierten politischen Kommunikation und der Ausprägung politischer Einstellungen. Jugendliche, die Politik stark personalisiert wahrnehmen, weisen eine niedrige informatorische Mobilisierung, ein hochsignifikant stärkeres Gefühl der Durchschaubarkeit von Politik vor allem während der heißen Wahlkampfphase, eine hochsignifikant niedrigere externe politische Wirksamkeit und einen starken Zynismus auf.

Die Kommunikation über Politik findet mit den Eltern deutlich häufiger statt (im Mittel rund 3,0 Punkte) als mit Freunden (rund 2,4 Punkte), was noch nichts über die Wirksamkeit dieser Agenturen der politischen Sozialisation aussagt. Zur Frage der Funktionalität der verschiedenen Sozialisationsagenturen für das Verstehen von Politik wurde wie folgt operationalisiert: „Die Medien (beziehungsweise Eltern, Schule, Freunde) helfen mir gut, damit ich verstehen kann, was in der Politik vor sich geht." Die den Massenmedien zugeschriebene Funktionalität zum Verstehen des politischen Geschehens fällt etwas höher aus als die den Eltern zugeschriebene, aber viel höher als die der Schule und den Freunden beigemessene. In den verschiedenen Tranchen stimmten rund 26 Prozent der Aussage voll zu, dass Medien gut hilfreich seien, weitere 45 Prozent stimmten eher zu; der Mittelwert auf einer fünfteiligen Skala liegt bei 3,7. Bei der abgefragten Funktionalität der Schule zeigten sich erhebliche Unterschiede nach Untersuchungsland. Deutsche Befragte erreichten hier deutlich bessere Werte als österreichische oder schweizerische.

29 Ebenda, S. 587.

30 Maria Gerhards / Walter Klingler, Jugend und Medien: Fernsehen als Leitmedium. Entwicklungsphasen, Nutzung und Funktionen der Medien für Jugendliche, in: Media Perspektiven, 29. Jg. (1999), H. 11, S. 562 - 576, S. 564. 


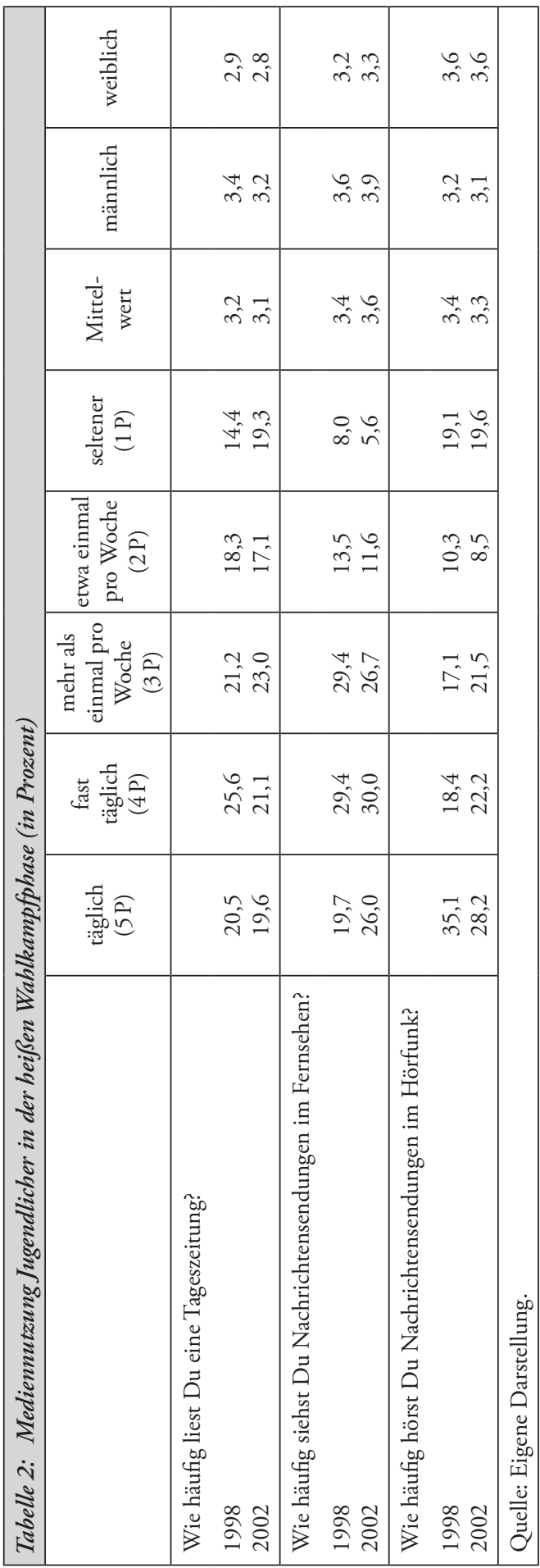




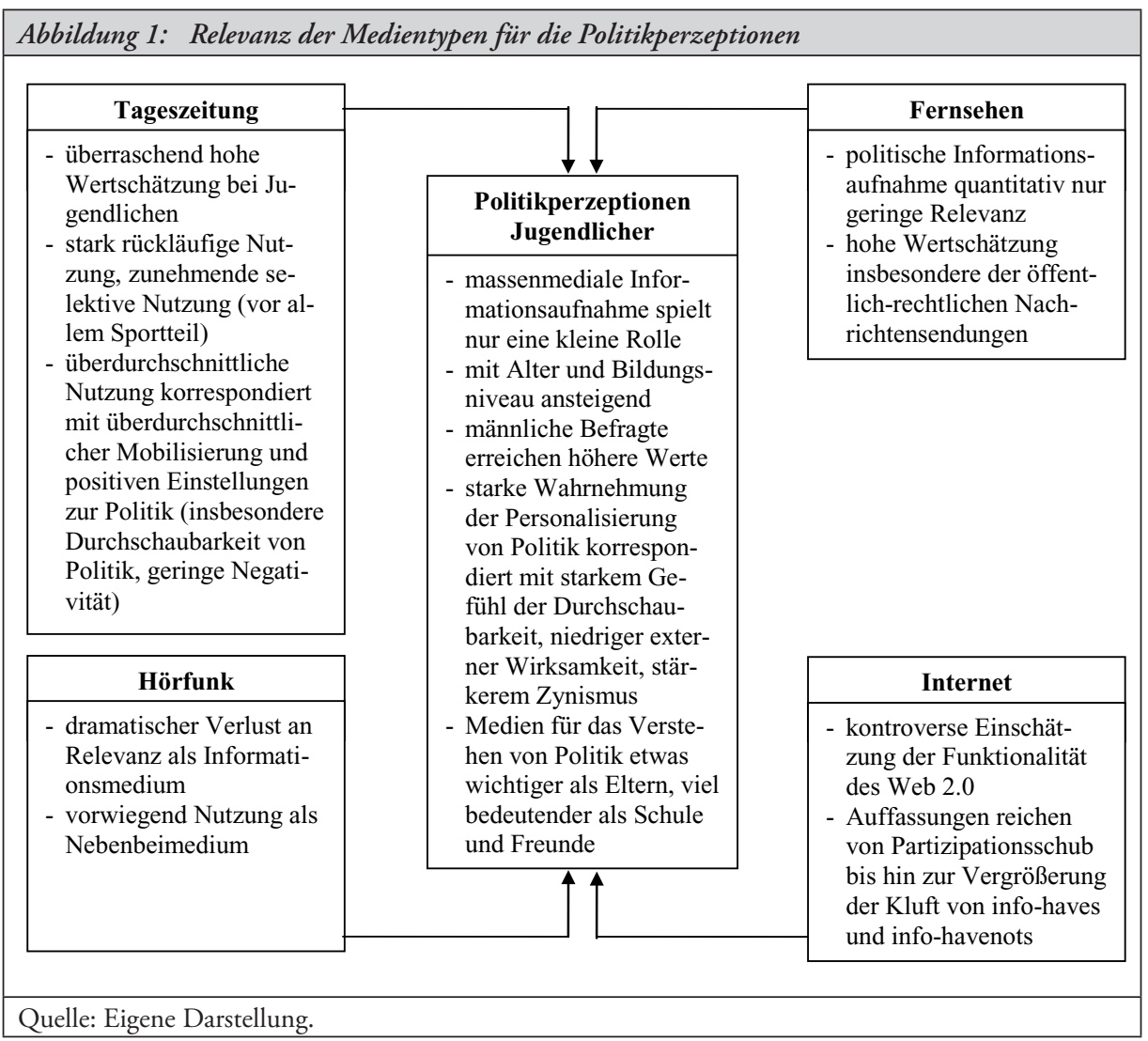

Insgesamt zeigt sich also, dass die geringe Nutzung politischer Informationen durch Jugendliche nicht einhergeht mit einer schlechten Bewertung der Leistung der Medien und auch nicht mit einer Negation ihrer Wirksamkeit für das Verstehen von Politik.

Die Debatte um das Web 2.0 mit seinen interaktiv angelegten Informationen wird auch im Hinblick auf die Rezeption politischer Informationen durch Jugendliche geführt. In der Literatur finden sich optimistische wie pessimistische Auffassungen, was die Effizienz des Internets betrifft, wobei die kritischen Stimmen überwiegen. Die Chance, dass das Web 2.0 zu einer Mobilisierung Jugendlicher führt, ist demnach eher unwahrscheinlich: „Das Internetangebot an politischen Informationen kann folglich für die politisch Interessierten als hervorragend bewertet werden, da es diese noch wissender machen kann, die Uninteressierten aber lässt es kalt. Es trägt potenziell sogar zu einer weiteren Differenzierung in infohaves and havenots bei." 31

31 Jürgen W. Falter, Demokratie im Internet, in: Gunnar Roters / Oliver Turecek / Walter Klingler (Hrsg.), Content im Internet, Trends und Perspektiven, Baden-Baden 2002, S. 19 - 22, S. 20. 


\section{Veränderung politischer Attitüden Jugendlicher unter Wablkampfbedingungen}

Die Antworten der befragten Jugendlichen stellen sich im Längsschnitt als recht stabil dar, wenngleich bei einigen Items eine größere Dynamik gegeben ist und auch Unterschiede zwischen den einzelnen Wahlkämpfen ersichtlich sind. 2002 war insbesondere die Stabilität der beabsichtigen Teilnahme an der Wahl und der parteipolitischen Präferenzen deutlich niedriger als vier Jahre zuvor, was vermutlich weniger Ausdruck von steigender Wählervolatilität als vielmehr ein Reflex der konkreten Kampagnen ist.

Hier wurde versucht, die während der heißen Wahlkampfphase und danach vorhandene Dynamik mit zwei Verfahren abzubilden: Erstens wurden die aggregierten Daten hinsichtlich ihrer Veränderungen untersucht. Es wurde also mit Hilfe von Varianzanalysen festgestellt, welche signifikanten Veränderungen unter der Berücksichtigung verschiedener Störvariablen (Kovariaten) während des Untersuchungszeitraums für das gesamte Sample festzustellen sind. Dabei muss allerdings berücksichtigt werden, dass es sich hierbei um Netto-Effekte handelt und dies Ergebnisse sehr unterschiedlicher Prozesse individuell steigender und fallender Zustimmungen zu den einzelnen Items sein können. Zweitens wurden, um die Brutto-Effekte auf individueller Ebene erfassen zu können, Clusterungen vorgenommen, was die heterogene Dynamik besser abbilden kann als die Analyse der Messwertwiederholungen für das gesamte Sample (vgl. Tabelle 3).

Von der Tendenz her kann für die Dynamik der aggregierten Daten während der Wahlkampfphase Folgendes festgestellt werden (auch wenn nicht alle Messwertveränderungen die strengen Anforderungen für ausreichende Signifikanz erreichen): Die informatorische Mobilisierung, das konventionelle Interesse, die Rezeption politischer Nachrichten und die Kommunikationsintensität mit den Eltern und Freunden steigen während des Wahlkampfs an, wobei dieser Anstieg auf den Kampagneneinfluss und auf Alterssteigerungen zurückzuführen ist. Insgesamt kann aber ein mobilisierender Einfluss auf Jugendliche nicht widerlegt werden, er ist vielmehr sehr wahrscheinlich. Keine eindeutige Zunahme ist bei der objektbezogenen Mobilisierung und beim prospektiven Engagement auszumachen. Die alternative kognitive Mobilisierung („Ich interessiere mich dafür, wie unsere Gesellschaft wichtige Probleme löst") ist sogar rückläufig - trotz der fördernden Einflüsse der während der Befragung stattfindenden Alterssteigerungen. Die Spaltung der politischen Mobilisierung Jugendlicher in eine konventionelle und eine alternative ist also auch unter Wahlkampfbedingungen deutlich festzustellen, und zwar auf eine paradoxe Weise: Während im Längsschnitt die konventionelle Mobilisierung, also das Interesse an der politics-Perspektive ab-, und die alternative, das Interesse an den policies, zunimmt, verhält sich dies im Wahlkampf genau umgekehrt. Wahlkampf zieht das Interesse Jugendlicher auf die politics-Dimension und weg von den policies. Die Auseinandersetzung mit der Programmatik wird eher verhindert. Dies dürfte auch mit der unter dem Begriff der Amerikanisierung beschriebenen Entideologisierung zu tun haben. Die Art der Wahlkampfpräsentation mit Komplexitätsreduktion durch Personalisierung konkretisiert sich in einem gestiegenen Gefühl, Politik durchschauen zu können, wobei hier wiederum Alterseffekte mitbeteiligt sind. Die interne Wirksamkeit, also die selbst wahrgenommene Kompetenz zur Einflussnahme auf Politik, bessert sich nicht. Die Wahrnehmung der Responsivität des politischen Systems nimmt während der Kampagnen zu, um in der Nachwahlkampfzeit deutlich abzufallen. Der insgesamt überraschend schwach ausgeprägte politische Zynismus reagiert nicht auf Wahlkampf. 


\begin{tabular}{|c|c|c|c|c|c|c|c|c|}
\hline \multirow{8}{*}{\multicolumn{2}{|c|}{ 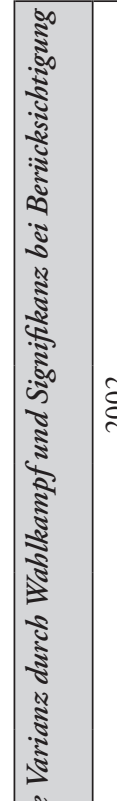 }} & \multicolumn{2}{|c|}{ : } & 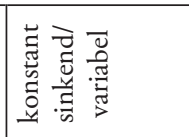 & 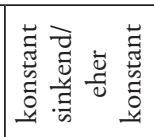 & 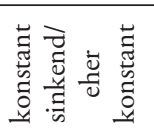 & 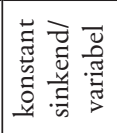 & 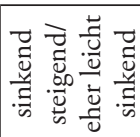 \\
\hline & & \multicolumn{2}{|c|}{ A } & 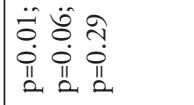 & 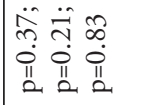 & 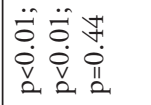 & 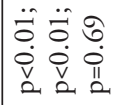 & 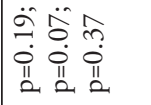 \\
\hline & & \multicolumn{2}{|c|}{ 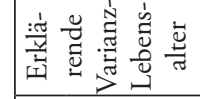 } & ¿ & $\ddot{0}$ & $\stackrel{0}{0}$ & $\stackrel{0}{0}$ & $\underset{O}{0}$ \\
\hline & & \multicolumn{2}{|c|}{ 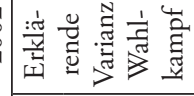 } & $\stackrel{\sim}{\tilde{c}}$ & $\underset{\text { กิ }}{\tilde{o}}$ & $\stackrel{\circ}{\because}$ & స̃. & $\underset{0}{0}$ \\
\hline & & \multicolumn{2}{|c|}{ 运 $\hat{F} \hat{H}$} & $\begin{array}{l}\overrightarrow{8} \\
\dot{0}\end{array}$ & $\stackrel{*}{*}$ & $\stackrel{*}{*}$ & $\stackrel{*}{*}$ & $\stackrel{*}{*}$ \\
\hline & & \multirow{3}{*}{ 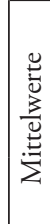 } & $\stackrel{m}{H}$ & $\overrightarrow{\mathrm{N}}$ & $\begin{array}{l}\stackrel{\leftrightarrow}{\sim} \\
\text { in }\end{array}$ & $\begin{array}{l}\text { } \\
i \\
i\end{array}$ & 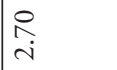 & $\begin{array}{l}\stackrel{\sim}{n} \\
\tilde{n}\end{array}$ \\
\hline & & & $\stackrel{\sim}{\mathrm{F}}$ & $\underset{\mathrm{d}}{\mathrm{i}}$ & $\begin{array}{l}n \\
\tilde{r}\end{array}$ & oे. & $\hat{\text { ô. }}$ & ले \\
\hline & & & $F$ & $\hat{\AA}$ & $\stackrel{\text { }}{\text { i }}$ & $\begin{array}{l}\hat{\theta} \\
\dot{r}\end{array}$ & $\begin{array}{l}\hat{\partial} \\
\dot{r}\end{array}$ & $\begin{array}{l}\tilde{n} \\
\tilde{n}\end{array}$ \\
\hline 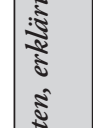 & \multirow{8}{*}{$\stackrel{\infty}{\check{\beth}}$} & \multicolumn{2}{|c|}{ 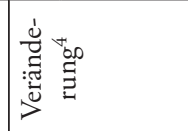 } & 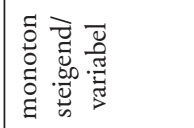 & 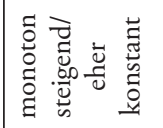 & 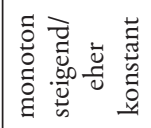 & 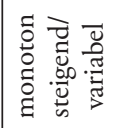 & 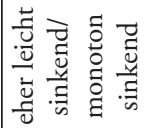 \\
\hline 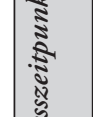 & & \multicolumn{2}{|c|}{ 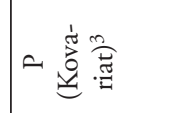 } & 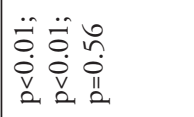 & 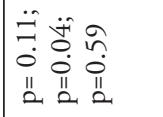 & 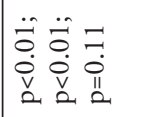 & 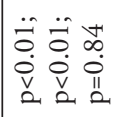 & 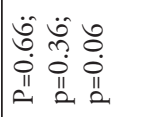 \\
\hline $\begin{array}{c}3 \\
\frac{\pi}{2}\end{array}$ & & \multicolumn{2}{|c|}{ 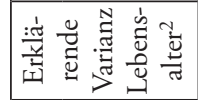 } & $\tilde{O}_{0}$ & $\stackrel{1}{0}$ & $\stackrel{\sigma}{0}$ & $\ddot{0}$ & $\underset{0}{0}$ \\
\hline 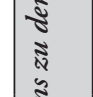 & & \multicolumn{2}{|c|}{ 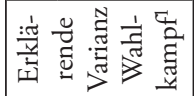 } & $\stackrel{\leftrightarrow}{0}$ & $\stackrel{0}{\circ}$ & $\stackrel{⿱ ⺌ 冖}{0}$ & $\stackrel{m}{0}$ & $\underset{0}{0}$ \\
\hline $\begin{array}{l} \pm \\
\pm \\
\pm\end{array}$ & & \multicolumn{2}{|c|}{ î̉ } & $\stackrel{*}{*}$ & $\stackrel{*}{*}$ & $\stackrel{*}{*}$ & $* *$ & $\stackrel{*}{*}$ \\
\hline 8ू. & & \multirow{3}{*}{$\frac{0}{0}$} & $\stackrel{m}{H}$ & $\begin{array}{l}\hat{n} \\
i\end{array}$ & $\begin{array}{l}\partial \\
\text { iे }\end{array}$ & $\begin{array}{l}\infty \\
\dot{n}\end{array}$ & $\stackrel{\leftrightarrow}{\grave{i}}$ & $\begin{array}{l}\infty \\
m \\
m\end{array}$ \\
\hline है & & & $\stackrel{F}{F}$ & $\begin{array}{l}\stackrel{i}{+} \\
\text { i }\end{array}$ & $\begin{array}{l}\vec{\infty} \\
i\end{array}$ & $\begin{array}{l}\text { के } \\
\text { ì }\end{array}$ & $\begin{array}{l}\infty \\
\infty \\
\stackrel{i}{i}\end{array}$ & $\begin{array}{l}\stackrel{0}{+} \\
\text { r. }\end{array}$ \\
\hline ปे & & & $F$ & $\underset{\tilde{N}}{\tilde{N}}$ & $\begin{array}{l}\underset{\mathrm{S}}{\mathrm{i}} \\
\mathrm{i}\end{array}$ & $\begin{array}{l}\vec{\infty} \\
\dot{N}\end{array}$ & $\begin{array}{l}\infty \\
i \\
i\end{array}$ & 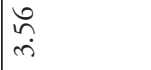 \\
\hline 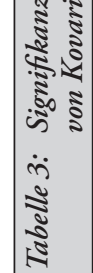 & & & & 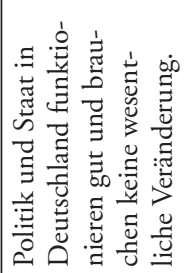 & 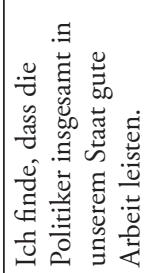 & 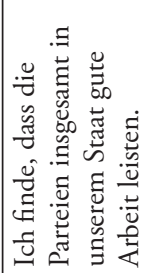 & 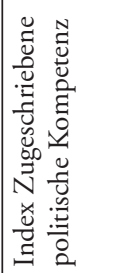 & 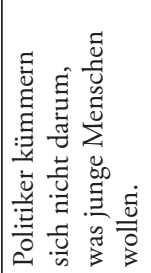 \\
\hline
\end{tabular}




\begin{tabular}{|c|c|c|c|c|}
\hline 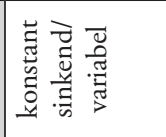 & 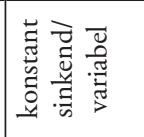 & 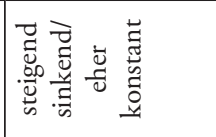 & 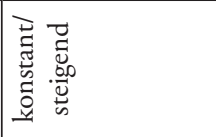 & 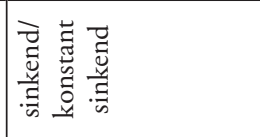 \\
\hline 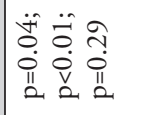 & 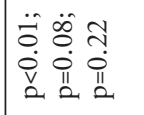 & 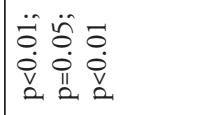 & 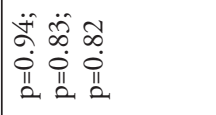 & 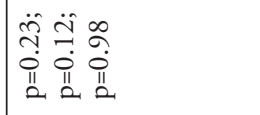 \\
\hline ¿্口. & $\begin{array}{l}8 \\
0 \\
0\end{array}$ & $\ddot{0}$ & $\stackrel{\Delta}{*}$ & $\begin{array}{l}n \\
0 \\
0\end{array}$ \\
\hline $\begin{array}{l}n \\
o \\
o\end{array}$ & $\begin{array}{l}n \\
0 \\
0\end{array}$ & $\underset{\delta}{0}$ & $\overrightarrow{0}$ & 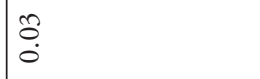 \\
\hline * & $\begin{array}{l}\overrightarrow{8} \\
\dot{v}\end{array}$ & $\begin{array}{l}\vec{\Xi} \\
\dot{v}\end{array}$ & $\stackrel{\dot{g}}{\dot{g}}$ & $*$ \\
\hline $\begin{array}{c}\infty \\
\stackrel{\sim}{i}\end{array}$ & 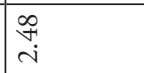 & $\frac{m}{i}$ & $\begin{array}{l}\hat{N} \\
\dot{n}\end{array}$ & $\overrightarrow{\dot{\omega}}$ \\
\hline 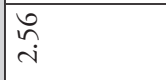 & 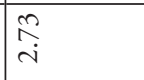 & $\begin{array}{l}\hat{i} \\
\text { ì }\end{array}$ & \begin{tabular}{|l}
$\alpha$ \\
$\infty$ \\
$\infty$ \\
$\dot{m}$
\end{tabular} & $\begin{array}{l}\text { D. } \\
\text { r. }\end{array}$ \\
\hline $\begin{array}{l}\text { तु } \\
\text { i } \\
\end{array}$ & \begin{tabular}{|l}
$\infty$ \\
0 \\
$i$ \\
\end{tabular} & $\overrightarrow{\vec{i}}$ & 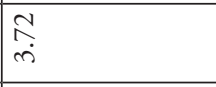 & $\begin{array}{l}\stackrel{+}{ } \\
\underset{j}{*}\end{array}$ \\
\hline 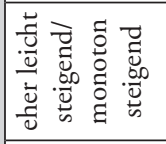 & 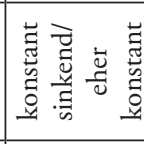 & 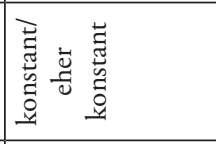 & 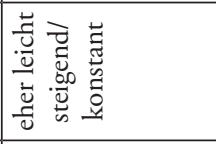 & 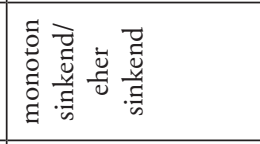 \\
\hline 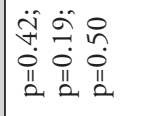 & 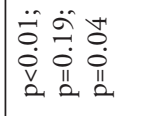 & 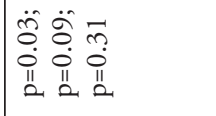 & 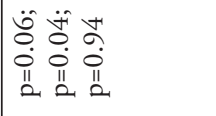 & 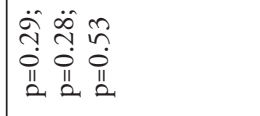 \\
\hline $\begin{array}{l}n \\
\dot{o}\end{array}$ & $\stackrel{8}{0}$ & $\stackrel{0}{0}$ & $\stackrel{+}{3}$ & 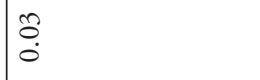 \\
\hline 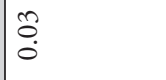 & $\stackrel{0}{0}$ & $\stackrel{8}{0}$ & $\vec{\sigma}$ & 菅 \\
\hline$\stackrel{*}{*}$ & $\begin{array}{l}\vec{\partial} \\
\dot{0} \\
\dot{v}\end{array}$ & $\begin{array}{l}\infty \\
\stackrel{0}{\circ} \\
\stackrel{0}{0}\end{array}$ & $\stackrel{\dot{a}}{=}$ & $\stackrel{*}{*}$ \\
\hline 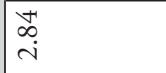 & $\begin{array}{l}\hat{\sigma} \\
i\end{array}$ & $\underset{i}{\stackrel{H}{i}}$ & \begin{tabular}{l|}
$\infty$ \\
$\dot{\omega}$ \\
$\dot{n}$
\end{tabular} & $\begin{array}{l}\vec{n} \\
\tilde{n}\end{array}$ \\
\hline 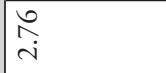 & $\begin{array}{l}\infty \\
\stackrel{\infty}{ } \\
i\end{array}$ & $\underset{i}{\stackrel{i}{i}}$ & $\overrightarrow{\dot{m}}$ & $\vec{m}$ \\
\hline $\begin{array}{l}\tilde{\sigma} \\
i \\
i\end{array}$ & $\begin{array}{l}\vec{\infty} \\
i\end{array}$ & $\stackrel{\vec{H}}{\vec{i}}$ & $\begin{array}{l}\hat{\sigma} \\
\dot{r}\end{array}$ & 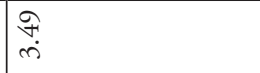 \\
\hline 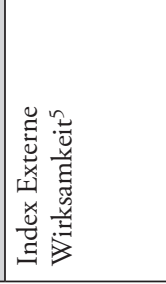 & 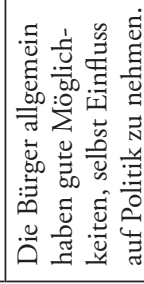 & 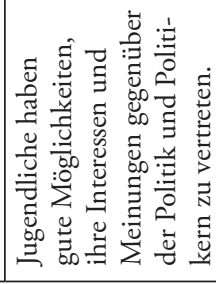 & 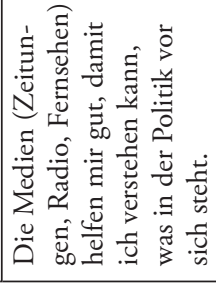 & 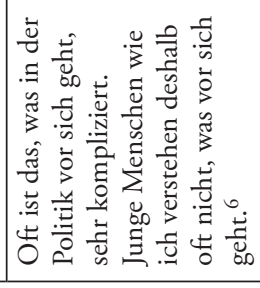 \\
\hline
\end{tabular}




\begin{tabular}{|c|c|c|c|c|}
\hline & 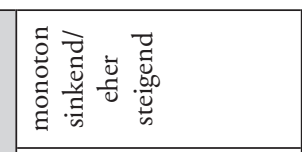 & 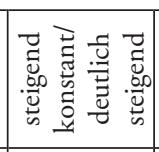 & 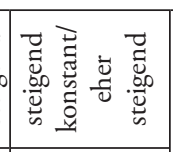 & \\
\hline & 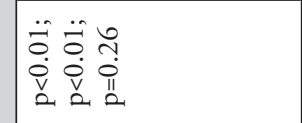 & 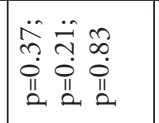 & 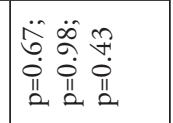 & \\
\hline & ठ̊. & $=$ & $\stackrel{n}{0}$ & \\
\hline & $\stackrel{n}{\circ}$ & $\stackrel{1}{0}$ & $\dot{\leftrightarrow}$ & \\
\hline & * & $*$ & $*$ & :ٌ \\
\hline & $\underset{n}{n}$ & $\begin{array}{ll}0 \\
m \\
m\end{array}$ & $\begin{array}{l}\tilde{N} \\
\tilde{n}\end{array}$ & 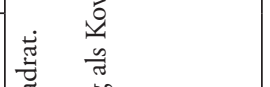 \\
\hline & $\underset{\dot{m}}{\vec{n}}$ & $\stackrel{\sim}{m}$ & $\stackrel{\infty}{m}$ & 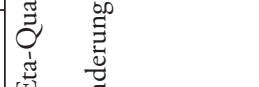 \\
\hline & 官 & $\begin{array}{l}\stackrel{\imath}{i} \\
i\end{array}$ & $\begin{array}{l}\stackrel{\infty}{i} \\
i\end{array}$ & \\
\hline & 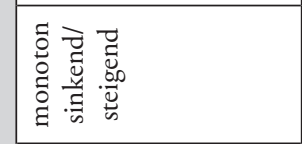 & 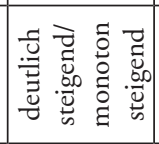 & 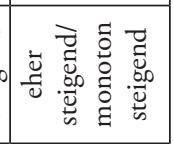 & 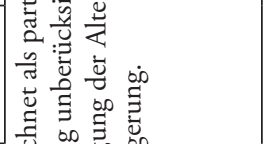 \\
\hline & 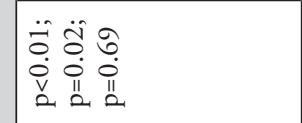 & 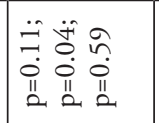 & 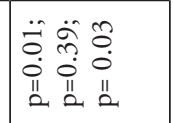 & 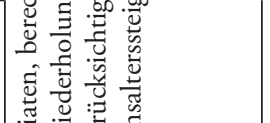 \\
\hline & $\begin{array}{l}n \\
o \\
0 \\
0\end{array}$ & $\ddot{0}$ & oे & 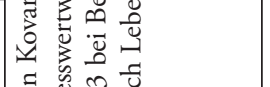 \\
\hline & $\stackrel{m}{0}$ & $\stackrel{m}{0}$ & $\stackrel{0}{0}$ & 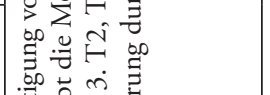 \\
\hline & * & $*$ & * & 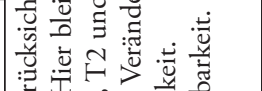 \\
\hline & 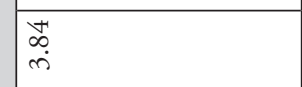 & $\overrightarrow{\tilde{n}}$ & $\vec{m}$ & 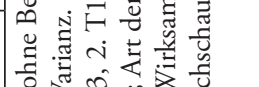 \\
\hline & $\begin{array}{l}\text { ஓे } \\
\dot{m}\end{array}$ & $\stackrel{\uplus}{m}$ & $\begin{array}{l}m \\
\dot{m}\end{array}$ & 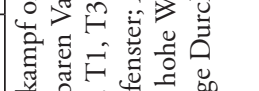 \\
\hline & $\underset{+}{\stackrel{H}{*}}$ & $\begin{array}{l}\infty \\
\infty \\
i\end{array}$ & $\hat{i}$ & 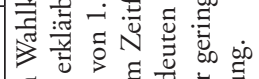 \\
\hline & 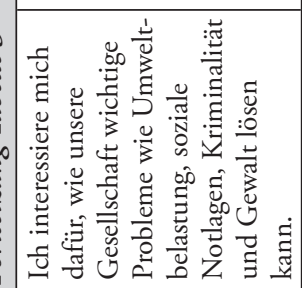 & 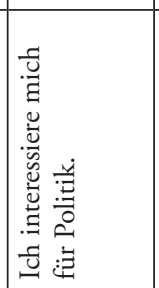 & 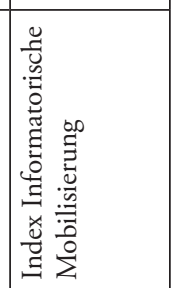 & 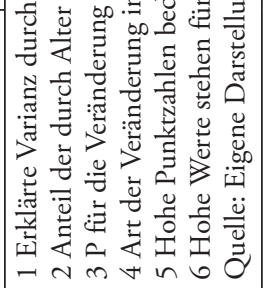 \\
\hline
\end{tabular}


Die von den Parteien dem Wahlkampf zugeschriebenen Leistungen der Mobilisierung werden erfüllt, was sich deutlich an der Zunahme der Wahlabsicht und der Existenz parteipolitischer Präferenzen zeigt. Aber auch die mittelfristige Dysfunktionalität im Sinne einer rückläufigen Legitimation zeigt sich. Dies ergibt sich aus der während des Wahlkampfs sinkenden externen Wirksamkeit, vor allem aber - zumindest für 2002 - aus dem Einbruch der Systemunterstützung nach der Wahl. Wahlkampf generiert Erwartungen, die die Politik nicht einzulösen in der Lage ist.

Die Analyse der Brutto-Effekte im Rahmen einer Clusterung ergibt, dass bei etwa einem Drittel der Jugendlichen während des Wahlkampfs erhebliche Veränderungen ihrer Mobilisierung und ihrer Einstellungen zu verzeichnen sind, die zum Teil aber nach dem Wahlkampf wieder kompensiert werden. Mehr Jugendliche erfahren durch Wahlkampf eine Verstärkung ihrer Mobilisierung als eine Schwächung. Bei sechs bis acht Prozent ist ein persistenter Rückgang der Mobilisierung festzustellen. Gleichermaßen ist während des Wahlkampfs häufiger eine Verbesserung der politischen Einstellungen als eine Verschlechterung zu sehen, wobei 2002 nach der Wahl eine massive zusätzliche politische Entfremdung eintrat. Dies ist besonders häufig bei Jugendlichen der Fall, die nach dem Wahlkampf eine zusätzliche Mobilisierung aufweisen, die offenbar zu verstärkten Enttäuschungen führt. Für etwa jeden siebten Jugendlichen hat Wahlkampf eine relativ dauerhafte dissoziative Wirkung, ist also aus der Perspektive des politisch-administrativen Systems dysfunktional.

Auch diese Analyse zeigt, dass Wahlkampf bei Jugendlichen eine Aktivation gegenüber Politik eher fördert als hemmt, dass aber nach der Wahl bei einem relativ großen Teil eine Verschlechterung ihrer politischen Einstellungen eintritt, die vermutlich auf Enttäuschungen bei höherer Aufmerksamkeit zurückzuführen sind. Die These, wonach Wahlkampf keine Wirkung auf Jugendliche hat, also die Neutralitätsthese, kann folglich eindeutig verworfen werden.

\begin{tabular}{|c|c|c|}
\hline \multicolumn{3}{|c|}{ Abbildung 2: Wablkampf und seine Wirkungen auf Jugendliche } \\
\hline & Wahlkampf & \\
\hline fördert & hat keine Effekte auf & reduziert \\
\hline $\begin{array}{l}\text { - die informatorische } \\
\text { Mobilisierung } \\
\text { - das Interesse am Ge- } \\
\text { schehen auf der politi- } \\
\text { schen Bühne (politics) } \\
\text { - die Rezeption politi- } \\
\text { scher Nachrichten } \\
\text { - die Kommunikationsin- } \\
\text { tensität mit Eltern und } \\
\text { Freunden } \\
\text { - das Gefühl der Durch- } \\
\text { schaubarkeit von Politik } \\
\text { - das Gefühl der } \\
\text { Responsivität des politi- } \\
\text { schen Systems }\end{array}$ & $\begin{array}{l}\text { - das Interesse an der } \\
\text { Lösung drängender } \\
\text { Probleme (policy) } \\
\text { - die interne Wirksamkeit } \\
\text { (Gefühl, aufgrund eige- } \\
\text { ner Fähigkeit Einfluss } \\
\text { nehmen zu können) } \\
\text { - den politischer Zynis- } \\
\text { mus }\end{array}$ & $\begin{array}{l}\text { - nach Ende des Wahl- } \\
\text { kampfs: das Gefühl der } \\
\text { Responsivität des politi- } \\
\text { schen Systems } \\
\text { - möglicherweise das } \\
\text { Systemvertrauen }\end{array}$ \\
\hline
\end{tabular}




\section{Empfehlungen für die Gestaltung von Wahlkämpfen}

Die Ergebnisse zeigen, dass Anstrengungen erforderlich sind, um die von Jugendlichen ausgehende Legitimation für das politisch-administrative System und für die Prinzipien einer repräsentativ-parlamentarischen Demokratie zu sichern; dies gilt umso mehr, als ein Verlust an Legitimation nur sehr mühsam wieder kompensiert werden kann.

Die Perzeption von Wahlkampf stellt sich als ein hochkomplexes, hochvariables Geschehen dar, bei denen Geschlecht, Alter und Bildungsniveau eine erhebliche Rolle spielen, was allgemeingültige Aussagen problematisch erscheinen lässt. Trotz dieser großen Individualität sind folgende Tendenzen offensichtlich:

(1) Wahlkampf verstärkt die Spaltung der kognitiven politischen Mobilisierung Jugendlicher in eine konventionelle, die die politics-Perspektive betrifft, und eine alternative, die policy-Aspekte fokussiert, in problematischer Weise. Kurzfristig steigt unter Wahlkampfbedingungen die konventionelle Mobilisierung von niedrigem Niveau kommend an, wie dies von den Wahlkampfstrategen ja auch intendiert ist. Da Jugendliche der Politischen Kultur mit Theatralität und Inszenierung skeptisch gegenüber stehen, liefert diese Aktivation Ressourcen, die nach Wahlkampfende eine Verschlechterung politischer Einstellungen nach sich ziehen könnte. Damit steigt die Rezeption von Negativität und Personalisierung, für die junge Menschen aufgrund der noch nicht weit entwickelten alternativen Schemata und Heuristiken besonders empfänglich sind. Hierin ist ein ernstes Dilemma zu sehen. Wahlkampf gelingt es kaum, Jugendliche auf die politischen Inhalte hin zu mobilisieren. Dies wird sogar eher behindert und damit eine Menge möglicher Involvierung verschenkt. Genau dies wäre aber zur Sicherung stabiler Legitimation sinnvoll und wünschenswert. $\mathrm{Zu}$ empfehlen ist, Jugendlichen die differenzierte Auseinandersetzung mit politischen Issues in ihrer Kontroversalität im Wahlkampf stärker zu ermöglichen. Dies könnte flankiert werden durch Anstrengungen im Bereich der schulischen und außerschulischen politischen Bildungsarbeit. Inwiefern Parteien und Politik zu einer solchen differenzierten Kommunikationsstrategie bereit und in der Lage sind, ist fraglich, wobei es hierzu in der Literatur nicht nur skeptische Stimmen gibt. Vorstellbar wären auch entsprechende Angebote in den von Jugendlichen genutzten Massenmedien und dem Internet, wenngleich in Bezug auf die Möglichkeiten des Internets - auch im Sinne des Web 2.0 - jüngst einige Ernüchterung eingetreten ist.

(2) Wahlkampf liefert durch sein Bemühen um wenig komplexe Argumentation Ressourcen, die bei Jugendlichen zu einer wachsenden Empfindung führen, politisches Geschehen durchschauen zu können. Dies dürfte wohl eine Illusion sein, die nach Ende des Wahlkampfs - wenn die gesamte Komplexität von Politik wieder zum Vorschein kommt erlebt wird und so Entfremdung nähren könnte. Auch hieraus muss die Empfehlung abgeleitet werden, Politik Jugendlichen gegenüber nicht vorwiegend im Rahmen einfacher Botschaften, so genannter „idiot lights“, sondern differenziert zu vermitteln. Dies ist nur dann möglich, wenn durch flankierende Maßnahmen, wie oben dargestellt, die Kompetenz Jugendlicher zum Verstehen von Politik entsprechend erhöht wird.

(3) Wahlkampf führt bei Jugendlichen nicht zum Anwachsen interner und externer Wirksamkeit, denn sie erleben nicht, dass ihnen im Rahmen von Kampagnen Gehör geschenkt wird und sie sich in den Prozess einbringen können. Gerade dieses Erleben wäre aber zur Sicherung diffuser Unterstützung im Sinne Eastons erforderlich, wobei dies nicht mit einer Wirksamkeitsillusion im Sinne symbolischer Politik verwechselt werden darf. 
Notwendig wäre eine echte stärkere Diskursivität in der politischen Kommunikation unter Wahlkampfbedingungen.

Deutlich zu sehen ist, dass die Zeit nach Ende des Wahlkampfs eine heikle Phase ist, wenn der politische Alltag wieder im Mittelpunkt steht. In dieser Phase, das gilt zumindest eindeutig für die Bundestagswahl von 2002, verschlechtern sich bei einem erheblichen Teil der Jugendlichen die zentralen politischen Einstellungen - offenbar aus Enttäuschung darüber, dass nur wenige der von den Parteien in Aussicht gestellten Modernisierungen umgesetzt werden können. Dann sind besondere kommunikative Kraftanstrengungen im Rahmen von Politikvermittlung und auch der politischen Bildungsarbeit sinnvoll, um diesen Effekt abzumildern und Verständnis für die komplexe Funktionslogik von Politik zu vermitteln.

\title{
Erfolgsbedingungen neuer Parteien im Parteiensystem am Beispiel der Piratenpartei Deutschland
}

\author{
Oskar Niedermayer
}

Sieht man vom Sonderfall der PDS/Linkspartei nach der deutschen Vereinigung ab, so hat es in der gesamten Geschichte des bundesrepublikanischen Parteiensystems nur eine neu gegründete Partei geschafft, sich auf Bundesebene dauerhaft als relevante Partei zu etablieren: die Grünen. Bedenkt man, dass in den letzten beiden Jahrzehnten neben den im Bundestag vertretenen Parteien im Schnitt 20 weitere an den Bundestagswahlen teilgenommen haben, so ist dies nicht gerade viel, und es stellt sich die Frage nach den Erfolgsbedingungen neuer Parteien im deutschen Parteiensystem. Da in neuerer Zeit von allen Kleinstparteien die Piratenpartei Deutschland bei weitem die größte Aufmerksamkeit erfahren hat und sie bei der Bundestagswahl 2009 unter den parlamentarisch nicht repräsentierten Parteien das mit Abstand beste Ergebnis verbuchen konnte, soll dieser Frage am Beispiel der Piratenpartei nachgegangen werden.

\section{Erfolg und Erfolgsbedingungen neuer Parteien im Parteiensystem}

Der „Erfolg“ einer neuen Partei wird hier durch das sukzessive Überschreiten von mehreren Karrierestufen gemessen, die qualitative Veränderungen der Rolle einer Partei im Parteiensystem markieren. Diese Entwicklung als „Lebenszyklus“1 zu sehen, ist in der Parteienforschung nicht neu. Eines der bekanntesten Modelle ist das „lifespan“-Modell von Pedersen²,

1 Im Lebenszyklus einer Partei geht es natürlich nicht nur aufwärts, es gibt auch Misserfolge, das heißt das Zurückfallen auf eine niedrigere Karrierestufe, bis hin zum „Tod“, das heißt zur Auflösung.

2 Vgl. Mogens N. Pedersen, Towards a New Typology of Party Lifespans and Minor Parties, in: Scandinavian Political Studies, 5. Jg. (1982), H. 1, S. 1 - 16, S. 6 f. 